\title{
High-order Quasi-Curl Conforming functions for Multiplicative Calderón Preconditioning of the EFIE
}

\author{
Felipe Valdés $^{*(1)}$, Francesco P. Andriulli ${ }^{(2)}$, Kristof Cools $^{(3)}$, and Eric Michielssen ${ }^{(1)}$ \\ (1)Radiation Laboratory, University of Michigan at Ann Arbor \\ (2)Department of Electronics (DELEN), Politecnico di Torino \\ (3)Department of Information Technology (INTEC), Ghent University
}

\section{Introduction}

Method of moments (MoM)-based electric field integral equation (EFIE) solvers are widely used for analyzing time-harmonic electromagnetic scattering from perfect electrically conducting (PEC) surfaces. In the last decade, MoM-based EFIE solvers using high-order basis functions [1] have been shown to be more accurate as well as CPU and memory efficient than their zeroth-order predecessors leveraging Rao-WiltonGlisson (RWG) functions [2]. Due to the spectral properties of the EFIE operator [3], and their effects on the MoM interaction matrices, the use of Calderón identities for preconditioning the EFIE has been studied extensively. In this context, BuffaChristiansen (BC) basis functions [4] recently have been shown to permit a robust discretization of the Calderón preconditioned EFIE [5,6]. Unfortunately, the $\mathrm{BC}$ basis functions are zeroth-order in nature, and only can be used in conjunction with RWG basis functions. This limitation imposes a severe constraint on the accuracy and efficiency of present Calderón preconditioned EFIE solvers. In this paper, a new set of basis functions is presented, that extends the qualities of the (zeroth-order) $\mathrm{BC}$ functions to high-order.

\section{Background}

Consider a closed PEC surface $S$ residing in a homogeneous medium with electric permittivity $\epsilon$ and magnetic permeability $\mu$. The electric current $\boldsymbol{J}$ induced on $S$ by the time-harmonic incident electric field $\boldsymbol{E}_{i n c}$ satisfies the EFIE

$$
\mathcal{T}(\boldsymbol{J})=-\hat{\boldsymbol{n}}_{r} \times \boldsymbol{E}_{\text {inc }},
$$

Where the EFIE operator is defined as $\mathcal{T}(\boldsymbol{J})=\mathcal{T}_{s}(\boldsymbol{J})+\mathcal{T}_{h}(\boldsymbol{J})$, with

$$
\mathcal{T}_{s}(\boldsymbol{J})=\frac{i k}{4 \pi} \hat{\boldsymbol{n}}_{r} \times \int_{S} \frac{e^{i k\left|\boldsymbol{r}-\boldsymbol{r}^{\prime}\right|}}{\left|\boldsymbol{r}-\boldsymbol{r}^{\prime}\right|} \boldsymbol{J}\left(\boldsymbol{r}^{\prime}\right) d s^{\prime},
$$

and

$$
\mathcal{T}_{h}(\boldsymbol{J})=\frac{i}{4 \pi k} \hat{\boldsymbol{n}}_{r} \times \int_{S} \nabla^{\prime} \frac{e^{i k\left|\boldsymbol{r}-\boldsymbol{r}^{\prime}\right|}}{\left|\boldsymbol{r}-\boldsymbol{r}^{\prime}\right|} \nabla^{\prime} \cdot \boldsymbol{J}\left(\boldsymbol{r}^{\prime}\right) d s^{\prime} .
$$

Here $k=\omega \sqrt{\epsilon \mu}$ is the wave number, $\omega$ is the source's angular frequency, $i=\sqrt{-1}$, and $\hat{\boldsymbol{n}}_{r}$ is an outward directed unit vector normal to $S$ at observer $\boldsymbol{r} \in S$. To solve EFIE (1) by the MoM, $S$ is approximated by a mesh $S_{\delta}$ of planar or curvilinear triangles with minimum edge size $\delta$, and $\boldsymbol{J}(\boldsymbol{r})$ is approximated as $\boldsymbol{J}(\boldsymbol{r}) \approx \sum_{n=1}^{N} I_{n} \boldsymbol{f}_{n}(\boldsymbol{r})$ where $\boldsymbol{f}_{n}(\boldsymbol{r})$, $n=1, \ldots, N$ are order- $p$ div-conforming Graglia-Wilton-Peterson (GWP) basis functions [1]. To obtain the expansion coefficients $I_{n}$, the above expression for $\boldsymbol{J}(\boldsymbol{r})$ is substituted into (1) and the resulting equation is tested with curl-confirming basis functions $\hat{\boldsymbol{n}}_{r} \times \boldsymbol{f}_{n}(\boldsymbol{r})$, yielding the $N \times N$ MoM EFIE system

$$
\overline{\overline{\mathbf{Z}}} \overline{\mathbf{I}}=\overline{\mathbf{V}} \text {, }
$$


where $(\overline{\overline{\mathbf{Z}}})_{i, j}=\left\langle\hat{\boldsymbol{n}}_{r} \times \boldsymbol{f}_{i}, \mathcal{T}\left(\boldsymbol{f}_{j}\right)\right\rangle,(\overline{\mathbf{V}})_{i}=-\left\langle\hat{\boldsymbol{n}}_{r} \times \boldsymbol{f}_{i}, \hat{\boldsymbol{n}}_{r} \times \boldsymbol{E}_{\text {inc }}\right\rangle$, and $(\overline{\mathbf{I}})_{j}=I_{j}$. For large $N$, (4) only can be solved iteratively. Unfortunately, the spectral properties of the operator $\mathcal{T}$ [3] are such that the condition number of $\mathbf{Z}$ grows without bound as $\boldsymbol{J}(\boldsymbol{r})$ is better approximated ( $\delta \rightarrow 0$ and/or $p \rightarrow \infty$ ). This problem can be solved by using the selfregularizing properties of $\mathcal{T}$ [5-7] expressed by the Calderón identities. The Calderónpreconditioned EFIE reads

$$
\mathcal{T}^{2}(\boldsymbol{J})=-\mathcal{T}\left(\hat{\boldsymbol{n}}_{r} \times \boldsymbol{E}_{\text {inc }}\right) .
$$

Since all singular values of the operator $\mathcal{T}^{2}$ accumulate at $-1 / 4$, the discretization of (5) leads to a well-conditioned MoM system, independently of $\delta$ and $p$. Standard discretization techniques which are suitable for stand-alone operators like $\mathcal{T}$ cannot be used straightforwardly when dealing with products like $\mathcal{T}^{2}=\mathcal{T} \cdot \mathcal{T}$. On the one hand, the direct discretization of these products is not feasible since $\mathcal{T}\left(\boldsymbol{f}_{n}\right)$ is not available in closed form. On the other hand, when div/curl-conforming GWPs are used to discretize the source/test space of $\mathcal{T}$, the product of the discretized stand-alone operators would require the inversion of a mixed div/curl-conforming Gram matrix $(\overline{\overline{\mathbf{G}}})_{i, j}=\left\langle\hat{\boldsymbol{n}} \times \boldsymbol{f}_{i}, \boldsymbol{f}_{j}\right\rangle$, which is singular [4]. A fundamental feature of the product $\mathcal{T}^{2}=\mathcal{T}_{s}^{2}+\mathcal{T}_{s} \mathcal{T}_{h}+\mathcal{T}_{h} \mathcal{T}_{s}+\mathcal{T}_{h}^{2}$ is that $\mathcal{T}_{h}^{2}=0$. Any discretization technique for $\mathcal{T}^{2}$ must preserve this last identity, otherwise it will lead to a system that does not possess the spectral properties of the continuous operator $\mathcal{T}^{2}$. Several discretization techniques for $\mathcal{T}^{2}$ exist in the literature. Many of them call for the separate discretization of all terms in the operator product $\mathcal{T}^{2}$, this by means of an artificial annihilation of $\mathcal{T}_{h}^{2}$ and appropriate operational manipulations to compute the other three terms $[7,8]$. Even though these techniques have been shown to give satisfactory results in frequency-domain simulations, they no longer preserve their qualities when dealing with time-domain marching on time EFIEs [6]. A different kind of discretization technique that uses BC basis functions [4] $f^{B C}$ to systematically construct a multiplicative discretization of $\mathcal{T}^{2}$ was presented in [5]. This technique also was shown effective for dielectric structures [9] as well as for PEC structures in time-domain simulations [6]. When $\mathcal{T}^{2}$ is discretized using $\mathrm{BC}$ basis functions [5,6] the identity $\mathcal{T}_{h}^{2}=0$ is preserved upon discretization. Unfortunately, BC basis functions are zeroth-order in nature, and therefore can only be used with (zerothorder) RWG basis functions. In what follows, a new set of basis functions is described, that extends the properties of the $\mathrm{BC}$ functions to high-order for use in conjunction with GWP basis functions in the construction of high-order Calderón preconditioned EFIE solvers.

\section{Construction of the higher order quasi-curl conforming basis functions}

The effectiveness of the technique proposed in [5] for discretizing $\mathcal{T}^{2}$ relies on two important properties of the $\mathrm{BC}$ basis functions: a) they are comprised of linear combinations of div-conforming RWG basis functions defined on the mesh $S_{\delta / 2}$, a barycentric refinement of the original mesh $S_{\delta}[4,5,6]$, and resemble the curl-conforming basis functions_defined on the original (coarse) mesh $S_{\delta}$ leading to a well-conditioned Gram matrix $\left(\overline{\mathbf{G}}_{n f B C}\right)_{i, j}=\left\langle\hat{\boldsymbol{n}}_{r} \times \boldsymbol{f}_{i}, \boldsymbol{f}_{j}^{B C}\right\rangle$. b) The dimension of the solenoidal (divergencefree) subspace of the space spanned by the $\mathrm{BC}$ basis functions matches the dimension of the rotational (curl $\neq 0$ ) subspace of the space spanned by the curl-conforming RWG basis functions. As explained in [6], this property leads to the preservation of the identity $\mathcal{T}_{h}^{2}=0$ upon discretization of $\mathcal{T}^{2}$. 
The new high-order quasi-curl conforming basis functions are constructed such that: a) as a linear combination of order- $p$ div-conforming GWP basis functions defined on $S_{\delta / 2}$, they resemble the order- $p$ curl-conforming GWP basis functions defined on $S_{\delta}$. b) They span a space (denoted by $\widetilde{X}$ ) whose solenoidal subspace has the same dimension as the rotational subspace of the space spanned by the curl-conforming GWP basis functions defined on $S_{\delta}$.

Let $X$ be the space spanned by the $N$ order- $p$ div-conforming GWP basis functions defined on $S_{\delta} . X$ can be decomposed into a solenoidal subspace $X_{\text {sol }}$ and its complement (non-solenoidal) subspace $X_{\text {nonsol }}$ [10]. Elements in the basis of $X_{\text {sol }}$ will be referred as "Standard Loops", while those in the basis of $X_{\text {nonsol }}$, as "Standard Stars". On its curl-conforming counterpart, a similar decomposition holds: If $\hat{\boldsymbol{n}} \times X$ denotes the space spanned by the curl-conforming GWP basis functions, then " $\hat{\boldsymbol{n}} \times$ Standard Loops" span the irrotational subspace $\hat{\boldsymbol{n}} \times X_{\text {sol }}$, and " $\hat{\boldsymbol{n}} \times$ Standard Stars" span the rotational subspace $\hat{\boldsymbol{n}} \times X_{\text {nonsol }}$. Similarly, if $X^{B}$ denotes the space spanned by the $N^{B}$ order- $p$ divconforming GWP basis functions defined on $S_{\delta / 2}$, then "Barycentric Loops" span $X_{\text {sol }}^{B}$, "Barycentric Stars" span $X_{\text {nonsol }}^{B}$, and so on. With both spaces, $X$ and $X^{B}$ being decomposed in terms of "loops" and "stars", the elements in the solenoidal subspace of $\widetilde{X}$ are obtained as the columns of the matrix

$$
\overline{\overline{\mathbf{P}}}_{L}=\left(\overline{\overline{\mathbf{L}}}_{B}^{T} \cdot \overline{\overline{\mathbf{G}}}_{f f}^{B} \cdot \overline{\overline{\mathbf{L}}}_{B}\right)^{-1} \cdot\left(\overline{\overline{\mathbf{L}}}_{B}^{T} \cdot \overline{\overline{\mathbf{G}}}_{n f f}^{B} \cdot \overline{\overline{\mathbf{R}}}_{\overline{\mathbf{S}}}\right) .
$$

Similarly, the elements in the non-solenoidal subspace of $\widetilde{X}$ are obtained as the columns of the matrix

$$
\overline{\overline{\mathbf{P}}}_{S}=\left(\overline{\overline{\mathbf{S}}}_{B}^{T} \cdot \overline{\overline{\mathbf{G}}}_{f f}^{B} \cdot \overline{\overline{\mathbf{S}}}_{B}\right)^{-1} \cdot\left(\overline{\overline{\mathbf{S}}}_{B}^{T} \cdot \overline{\overline{\mathbf{G}}}_{n f f}^{B} \cdot \overline{\overline{\mathbf{R}}} \cdot \overline{\overline{\mathbf{L}}}_{S}\right)
$$

Where: $\overline{\overline{\mathbf{L}}}_{B}$ is the matrix whose columns are the "Barycentric loops", $\overline{\overline{\mathbf{S}}}_{B}$ is the matrix whose columns are the "Barycentric stars", $\mathbf{L}_{S}$ is the matrix whose columns are the "Stqndard loops", $\mathbf{S}_{S}$ is the matrix whose columns $\underline{\underline{B}}_{3}$ are the "Standard stars", $\left(\overline{\mathbf{G}}_{f f}\right)_{i, j}=\left\langle\boldsymbol{f}_{i}^{B}, \boldsymbol{f}_{j}^{B}\right\rangle$ is the barycentric Gram matrix, $\left(\overline{\mathbf{G}}_{n f f}\right)_{i, j}=\left\langle\hat{\boldsymbol{n}}_{r} \times \boldsymbol{f}_{i}^{B}, \boldsymbol{f}_{j}^{B}\right\rangle$ is the barycentric mixed Gram matrix, and $\mathbf{R}$ is the mapping from GWP basis functions defined on $S_{\delta}$ to GWP basis functions defined on $S_{\delta / 2}$. All matrices listed above are sparse, and their inverses well-conditioned. Formulas (6)-(7) implement nothing but the $L_{2}$ projection of the basis of $\hat{\boldsymbol{n}} \times X_{\text {nonsol }}$ onto $X_{\text {sol }}^{B}$ and $\hat{\boldsymbol{n}} \times X_{\text {sol }}$ onto $X_{\text {nonsol }}^{B}$, respectively.

\section{Numerical Results}

This section presents an example that demonstrates the effectiveness of the high-order quasi-curl conforming basis functions constructed here. Fig. 1 shows the error in bistatic RCS of a PEC sphere of radius $R=\lambda / 10$ illuminated by an $x$-polarized plane wave propagating in the $z$ direction. The RCS results are obtained for different orders and compared to the Mie series solution. Clearly, as the order increases, the error goes down. Table 1 shows the condition number of the EFIE and the Caderon-preconditioned EFIE for several orders and discretizations. For each order, the condition number of $\left(\mathcal{T}^{2}\right)_{\text {dis }}$ remains stable, independent of the discretization; whereas the condition number of $(\mathcal{T})_{d i s}$ grows as the number of unknowns increases. The condition number of the mixed Gram matrix between quasi-curl conforming and curl-conforming GWPs (for simplicity, here denoted as $\left.G_{n f B C}\right)$ is also shown. 


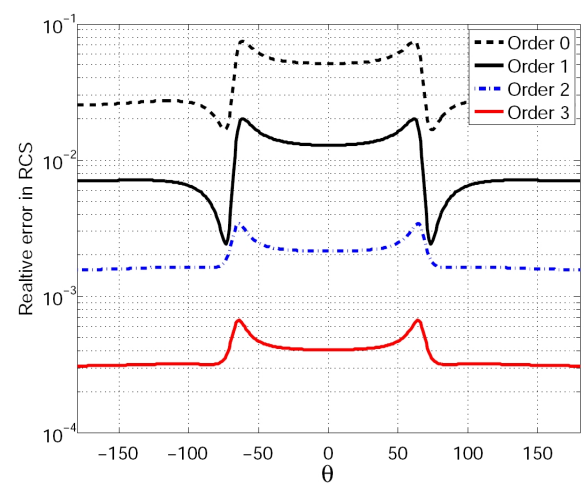

Fig. 1: Relative error in RCS in higher order Calderón-preconditioned EFIE.

\begin{tabular}{|c|c|c|c|c|}
\hline \multirow{4}{*}{ రై } & "\# unknowns & 160 & 510 & 1120 \\
\hline & $G_{n f B C}$ & 19.69 & 32.26 & 42.91 \\
\hline & $(\mathcal{T})_{d i s}$ & 408.47 & 2298.3 & 5476.3 \\
\hline & $\left(\mathcal{T}^{2}\right)_{d i s}$ & 22.03 & 25.83 & 33.17 \\
\hline \multirow{4}{*}{ סै } & \# unknowns & 336 & 1071 & 2352 \\
\hline & $G_{n f B C}$ & 55.84 & 80.9 & 88.73 \\
\hline & $(\mathcal{T})_{d i s}$ & 3434.2 & 20914 & 46557 \\
\hline & $\left(\mathcal{T}^{2}\right)_{d i s}$ & 62.69 & 93.5 & 83.63 \\
\hline \multirow{4}{*}{ שே } & \# unknowns & 576 & 1836 & \\
\hline & $G_{n f B C}$ & 141.6 & 206.1 & \\
\hline & $(\mathcal{T})_{d i s}$ & 29142 & 187130 & \\
\hline & $\left(\mathcal{T}^{2}\right)_{d i s}$ & 228.6 & 294.3 & \\
\hline
\end{tabular}

Table 1: Condition number for several orders and several mesh discretizations.

\section{References}

[1] R.D. Graglia, D.R. Wilton, and A.F. Peterson, "Higher order interpolatory vector bases for computational electromagnetics," IEEE Transactions on Antennas and Propagation, vol.45, no.3, pp.329-342, Mar 1997.

[2] S. M. Rao, D. R. Wilton, and A. W. Glisson, "Electromagnetic scattering by surfaces of arbitrary shape,” IEEE Trans. Antennas Propagat., vol. AP-30, pp. 409-418, May 1982.

[3] J.-C. Nedéléc, Acoustic and Electromagnetic Equations. New York: Springer-Verlag, 2000.

[4] A. Buffa and S. Christiansen, "A dual finite element complex on the barycentric refinement," Math. Comp., vol. 76, pp. 1743-1769, 2007.

[5] F.P. Andriulli, K. Cools, H. Bagci, F. Olyslager, A. Buffa, S. Christiansen, and E. Michielssen, "A Multiplicative Calderon Preconditioner for the Electric Field Integral Equation," IEEE Transactions on Antennas and Propagation, vol.56, no.8, pp.2398-2412, Aug. 2008.

[6] K. Cools, F.P. Andriulli, F. Olyslager, and E. Michielssen, "Time-Domain Calderón Identities and their Application to the Integral Equations analysis of Scattering by PEC objects Part I: Preconditioning", submitted to IEEE Transactions on Antennas and Propagation.

[7] H. Contopanagos, B. Dembart, M. Epton, J.J. Ottusch, V. Rokhlin, J.L. Visher, and S.M. Wandzura, "Well-conditioned boundary integral equations for three-dimensional electromagnetic scattering," IEEE Transactions on Antennas and Propagation, vol.50, no.12, pp. 1824-1830, Dec 2002.

[8] R.J. Adams, and N.J. Champagne, "A numerical implementation of a modified form of the electric field Integral equation," IEEE Transactions on Antennas and Propagation, vol.52, no.9, pp. 2262-2266, Sept. 2004

[9] F. Valdés, F.P. Andriulli, H. Bagci, and E. Michielssen, "On the discretization of single source integral equations for analyzing scattering from homogeneous penetrable objects," Antennas and Propagation Society International Symposium, 2008. AP-S 2008. IEEE, vol., no., pp.1-4, 5-11 July 2008.

[10] R.A. Wildman, and D.S. Weile, "An accurate broad-band method of moments using higher order basis functions and tree-loop decomposition," IEEE Transactions on Antennas and Propagation, vol.52, no.11, pp. 3005-3011, Nov. 2004 . 


\section{IEEE International Symposium on Antennas and Propagation and USNC/URSI National Radio Science Meeting}

Copyright and Reprint Permission: Abstracting is permitted with credit to the source. Libraries are permitted to photocopy beyond the limit of U.S. copyright law for private use of patrons those articles in this volume that carry a code at the bottom of the first page, provided the per-copy fee indicated in the code is paid through the Copyright Clearance Center, 222 Rosewood Drive, Danvers, MA 01923. For other copying, reprint, or replication permission, write to IEEE Copyrights Manager, IEEE Operations Center, 445 Hoes Lane, P.O. Box 1331, Piscataway, NJ, 08855-1331. All rights reserved. Copyright (C) 2009 by the Institute of Electrical and Electronics Engineers, Inc. Personal use of this material is permitted. However, permission to reprint/republish this material for advertising or promotional purposes or for creating new collective works for sale or distribution to servers or lists, or to reuse any copyrighted component of this work in other works must be obtained from the IEEE.

IEEE Catalog Number: CFP09APS-CDR

ISBN: 978-1-4244-3647-7

Library of Congress (Serialized): 90-640397

ISSN: 1522-3965

Support: If you have problems or questions related to the installation of this disc, please contact the 3WAIsmen at FAX: (818) 952-0183 or e-mail: wais3men@yahoo.com

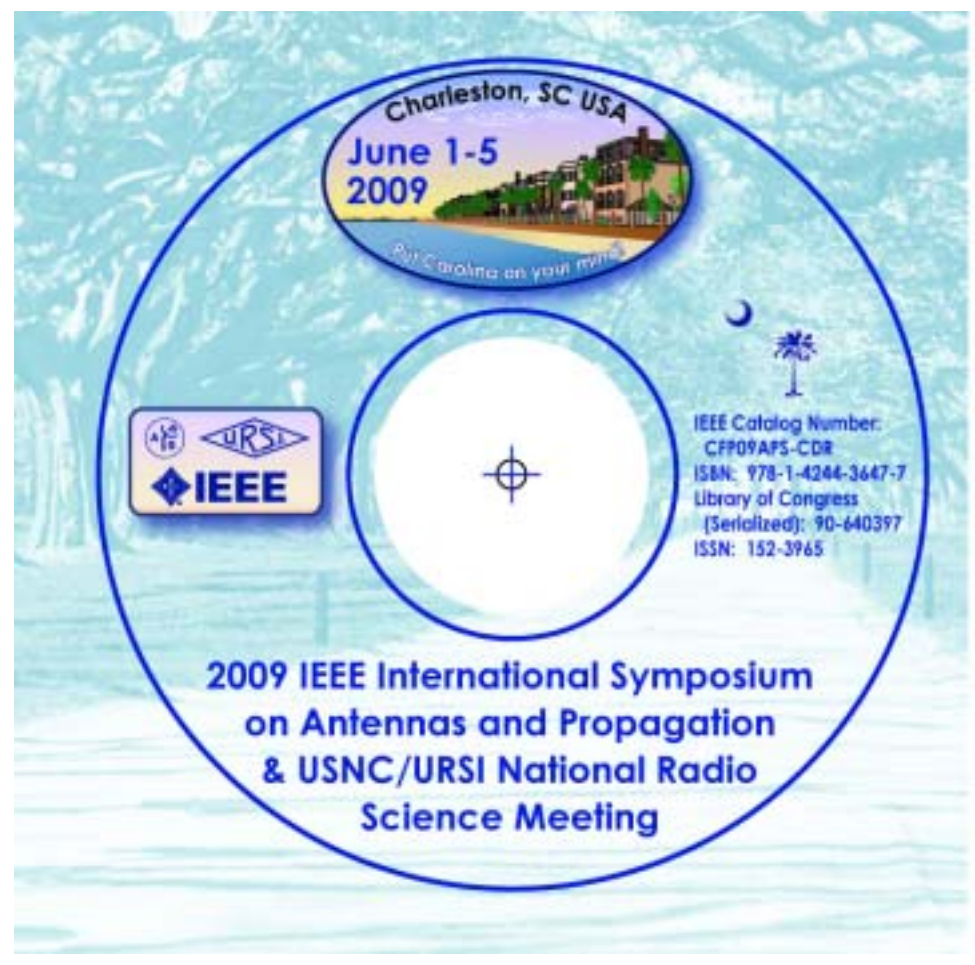

June 1-5, 2009

WA][s\}

Charleston, SC

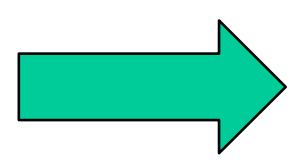

